\title{
The Oral Administration of Highly-Bioavailable Curcumin for One Year Has Clinical and Chondro- Protective Effects: A Randomized, Double-Blinded, Placebo-Controlled Prospective Study
}

\author{
Yasuaki Nakagawa, M.D., Koji Mori, Ph.D., Shigeru Yamada, M.D., Shogo Mukai, M.D., \\ Akiko Hirose, M.D., and Ryota Nakamura, M.D.
}

Purpose: The purpose of this study was to determine the clinical and chondroprotective efficacy and safety of orally administered Theracurmin in patients who underwent mosaicplasty for knee chondral or osteochondral diseases over 12 months of treatment. Methods: We enrolled 50 patients, older than 20 years of age, who underwent mosaicplasty for their knee joint diseases. Theracurmin at $180 \mathrm{mg}$ of curcumin per day or placebo was administered orally every day for 12 months. Because 7 patients dropped out of the study, 43 patients were examined; they included 14 men and 29 women and 24 right and 19 left knees. The mean operative age was 59.5 years (range, 24-84 years). We evaluated the Japanese Orthopaedic Association knee osteoarthritis score (JOA), visual analog scale (VAS), and Japanese Knee Osteoarthritis Measure (JKOM) as clinical symptoms; T2 mapping values using magnetic resonance imaging as an indication of the chondroprotective effect; and blood concentration of curcumin at $0,3,6$, and 12 months after the operations. We performed intraoperative acoustic evaluation of articular cartilage as a measure of chondroprotective effect during the operations and second-look arthroscopy. Results: The JOA, VAS and JKOM at 3, 6, and 12 months were significantly better than those during the preoperative period. However, the values of JOA, VAS and JKOM and T2 mapping were not significantly different between the Theracurmin and placebo groups. The blood concentration of curcumin in the Theracurmin group was significantly higher than that in the placebo group at 3, 6, and 12 months after the operations. Cartilage stiffness and surface roughness were significantly better in the Theracurmin group than in the placebo group at second-look arthroscopy. Conclusions: The oral administration of Theracurmin for 1 year demonstrated significantly better chondroprotective effects and no worse clinical effects and adverse events than the placebo. Level of Evidence: Level I, double-blinded, placebo-controlled, prospective study.

C urcumin is a polyphenol extracted from turmeric, which has been safely used in foods, such as curry for a long time. ${ }^{1}$ Curcumin is a promising therapeutic

From the Department of Orthopaedic Surgery, National Hospital Organization, Kyoto Medical Center (Y.N., S.Y., S.M., R.N.), Kyoto; Applied Medical Engineering Science, Yamaguchi University Graduate School of Medicine (K.M.), Yamaguchi; and Theravalues Corporation (A.H.), Tokyo, Japan.

The authors report the following potential conflicts of interest or sources of funding: Financially supported by Theravalues Corporation. Full ICMJE author disclosure forms are available for this article online, as supplementary material.

Received May 7, 2021; accepted October 23, 2021.

Corresponding author: Yasuaki Nakagawa, M.D., Department of Orthopaedic Surgery, National Hospital Organization Kyoto Medical Center, 1-1 Fukakusa Mukaihata-cho Fushimi-ku, Kyoto 612-8555, Japan. E-mail: yasu0202nakagawa@gmail.com

(C) 2021 THE AUTHORS. Published by Elsevier Inc. on behalf of the Arthroscopy Association of North America. This is an open access article under the CC BY-NC-ND license (http://creativecommons.org/licenses/by-nc-nd/4.0/). 2666-061X/21697

https://doi.org/10.1016/j.asmr.2021.10.016 food material because of its anti-inflammatory and antioxidative functions; it has long been used as an anti-inflammatory treatment in traditional Chinese and Ayurvedic medicine. ${ }^{1}$ Curcumin regulates various biochemical and molecular pathways by modulating several molecular targets, including transcription factors, cytokines, enzymes, and genes, that regulate cell proliferation or apoptosis. ${ }^{2}$ The anti-inflammatory effect of curcumin appears to be comparable with that of steroidal drugs and nonsteroidal anti-inflammatory drugs such as indomethacin and phenylbutazone. ${ }^{3}$ Studies have shown that the anti-inflammatory properties of curcumin are related to the suppression of prostaglandin synthesis through its effect on cyclooxygenase, ${ }^{4}$ a key enzyme responsible for the conversion of arachidonic acid to prostaglandins. Additionally, curcumin inhibited proteasome activity and induced apoptosis in human colon cancer cells in vitro and in vivo. ${ }^{5}$ Moreover, an important mechanism of action 
of curcumin is its inhibiton of NF-kB activation, ${ }^{6}$ which is a key event in the chronic inflammatory process. On the basis of these findings, curcumin is expected to be effective for multiple diseases related to chronic inflammation, including cancer, cardiovascular disease, metabolic syndrome, Alzheimer's disease, osteoarthritis, and other common diseases and aging conditions. ${ }^{1,2,7,8}$ Furthermore, curcumin can act as a potent inhibitor of the production of inflammatory and catabolic mediators by chondrocytes. ${ }^{7}$ Because osteoarthritis and related osteoarticular conditions of the synovial joints are characterized by inflammation, the biological effects of curcumin in joint tissues may facilitate the development of clinically safe, orally administered therapeutic agents for treating joint diseases. An articular chondrocyte study in humans ${ }^{9}$ clearly demonstrated that curcumin exerted antiapoptotic and anti-catabolic effects on interleukin (IL)-lb-stimulated articular chondrocytes. Therefore curcumin may exhibit novel therapeutic potential as an adjunct nutraceutical chondroprotective agent for treating osteoarthritis and related osteoarticular disorders. $^{9}$

However, the poor bioavailability of curcumin has been an obstacle to realizing its beneficial health effects because only a small amount of curcumin is absorbed via oral administration. ${ }^{10}$ To overcome the bioavailability problem, Sasaki previously developed a surfacecontrolled water-dispersible curcumin formulation named Theracurmin (Theravalues, Tokyo, Japan). ${ }^{11}$ They investigated the absorption efficacy of Theracurmin and compared with it that of curcumin powder. In rats, the area under the blood concentration-time curve after the oral administration of Theracurmin was more than 40-fold higher than that of curcumin powder. In healthy human volunteers, the area under the blood concentration-time curve of Theracurmin was 27-fold higher than that of curcumin powder. These findings demonstrate Theracurmin' $\mathrm{s}$ significantly higher bioavailability compared to currently available curcumin preparations.

Previously Nakagawa's research group, including a randomized, double-blinded, placebo-controlled, prospective clinical study ${ }^{12}$ and an open-labeled prospective study, ${ }^{13}$ demonstrated the anti-inflammatory effects of Theracurmin; however, few studies have reported the chondroprotective effects of Theracurmin and curcumin in a human clinical trial. Therefore the purpose of this study was to determine the clinical and chondroprotective efficacy and safety of orally administered Theracurmin in patients who underwent mosaicplasty for knee chondral or osteochondral diseases over 12 months of treatment. We hypothesized that Theracurmin ingestion for 12 months would improve the symptoms, functional abilities, and chondroprotective effects in patients with knee chondral or osteochondral diseases with no major side effects.

\section{Methods}

A randomized, double-blind, placebo-controlled, prospective clinical study was conducted to test our hypothesis in 2 treatment groups: Theracurmin and placebo. Randomization was performed after baseline tests and surgery and using a computer-generated numbers table without stratification at the start of the treatment period. The allocation (based on randomization) was performed by a person who was not involved in the current project. From August 2013 to February 2016, 50 patients over the age of twenty undergoing planned mosaicplasty for International Cartilage Repair Society (ICRS) grade 3 or 4 knee osteochondral lesions who underwent more than l year of follow-up were enrolled. Written informed consent was obtained from all subjects before participation. All procedures were reviewed and approved by the research ethics committee of our hospital, and this study was performed in accordance with the World Medical Association's Declaration of Helsinki. This study was registered to UMIN-CTR.

Fig 1 presents the flow chart of the study. Twenty-five patients were enrolled in the Theracurmin and placebo groups each. The drop-out cases due to serious adverse events are presented in Table 1 . There was 1 event in 1 case in the Theracurmin group and 6 events in 3 cases in the placebo group. Drop-outs occurred for 1 case in the Theracurmin group and 2 cases in the placebo group. The reasons for the drop outs were as follows: in

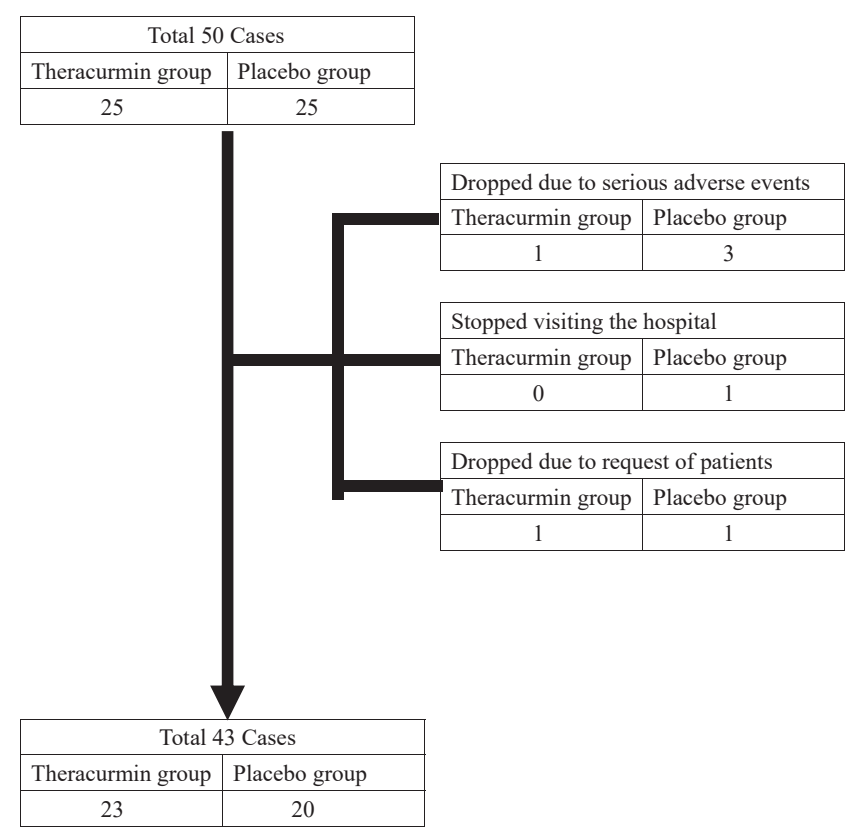

Fig 1. Flow chart of the study. 
Table 1. Serious Adverse Events (Drop-Out Cases) in the 2 Groups

\begin{tabular}{ll}
\hline \multicolumn{1}{c}{ Case No. } & \multicolumn{1}{c}{ Adverse Event } \\
\hline $\begin{array}{l}\text { Theracurmin group } \\
6\end{array}$ & Brain bleeding \\
Placebo group & \\
8 & Fracture and Infection \\
27 & Cholecystitis and Dermatitis \\
35 & Infection and Dermatitis \\
\hline
\end{tabular}

the Theracurmin group, anxiety of the patient that the patient might forget to take the drugs, and in the placebo group, the rejection of our outpatient rehabilitation in 1 case and discontinuation of visit to our outpatient clinic 8 months after mosaicplasty in another case. Therefore we included 43 patients (23 patients in the Theracurmin group and 20 in the placebo group) for further analysis.

According to our criteria, the candidates for mosaicplasty were patients with ICRS grade 3 or 4 osteochondral lesions in the knee who had received no effective conservative treatment for at least 3 months and whose operative age was more than 20 years. The exclusion criteria were the following: (1) severe renal dysfunction or dialysis, (2) severe liver dysfunction or liver cirrhosis, (3) severe cardiovascular diseases, (4) severe cerebrovascular dysfunction, (5) severe diabetes mellitus, (6) curcumin abuse patients, or (7) pregnancy.

According to our treatment protocol for osteochondral diseases in the knee joints, if the femorotibial angle is greater than $180^{\circ}$, high tibial osteotomy with mosaicplasty is performed. If the femorotibial angle is less than $168^{\circ}$, distal femoral varus osteotomy with mosaicplasty is performed. Therefore, if the femorotibial angle is between $168^{\circ}$ (valgus deformity) and $180^{\circ}$ (varus deformity), we perform mosaicplasty alone. The postoperative rehabilitation program was as follows: range-of-motion exercise was initiated on day 3 after mosaicplasty, partial weightbearing gait in week 2 or 3 , and full weightbearing gait in week 5 or 6 . The patient's donor site was either the lateral or medial side of the femoral trochlea.

Theracurmin or placebo was administered orally twice a day, for 52 weeks. Subjects in the Theracurmin group took six capsules of Theracurmin per day, which contained $180 \mathrm{mg}$ of curcumin. Similarly, subjects in the placebo group took 6 placebo capsules per day, which were of similar shape and color as the Theracurmin capsules; the capsules were primarily composed of starch, dextrin, and maltose. The subjects were requested to report the number of remaining capsules at their 1-, 2-, 3-, 4-, 5-, 6-, 8-, 10-, and 12 -month visits at our outpatient clinic for the compliance check.
Blood biochemistry parameters and blood concentration of curcumin were analyzed before the study and at 3, 6, and 12 months after mosaicplasty. We evaluated adverse events and the abnormal blood biochemistry values. If the same patient experienced 2 adverse events, we unblinded the treatment allocation (curcumin or placebo) and decided to exclude the patient from this study.

The patients' knee symptoms were evaluated at 0,3 , 6 , and 12 months according to the following criteria: the Japanese Knee Osteoarthritis Measure (JKOM), ${ }^{14}$ knee pain visual analog scale (VAS) included in the JKOM, and the Japanese Orthopaedic Association knee osteoarthritis score (JOA). ${ }^{12}$ The JKOM consists of 25 questions divided into four subcategories -pain and stiffness, condition in daily life, general activities, and health conditions- for patient self-assessment, it is based on the World Health Organization's International Classification of Functioning, Disability, and Health, validated in the same manner as the Western Ontario and McMaster Universities' Arthritis Index (WOMAC). ${ }^{15}$ The JOA scale evaluates four items: ability to walk (30 points), ability to climb up and down stairs ( 25 points), range of motion ( 35 points), and joint swelling (10 points). Each knee joint can achieve a maximum score of 100 points on the JOA scale.

To evaluate chondroprotective effects in the cartilage of the operated knee, we performed magnetic resonance imaging (MRI) of the operated knee, and arthroscopic acoustic evaluation. MRI was conducted before mosaicplasty and 3, 6, and 12 months after mosaicplasty, and $\mathrm{T} 2$ mapping values were used to evaluate cartilage characteristics in 6 areas: the medial or lateral femoral condyle, medial or lateral tibial plateau, patella, and trochlea. All MRI images were obtained on a clinical 1.5T unit (Achieva; Royal Philips, Amsterdam, the Netherlands) using a standard receiveonly 8-channel SENSE knee coil. Quantitative T2 mapping was performed using a multislice, multiecho, modified Carr Purcell Meiboom Gill pulse sequence, which uses interleaved slices and tailored refocusing pulses to minimize contribution from stimulated echoes. ${ }^{16,17}$ Quantitative T2 relaxation times or values were calculated by taking the natural logarithm of the signal decay curve in a selected region-of-interest (ROI). The ROIs were obtained in a standardized manner from the articular surface of the cartilage to the cartilage-bone interface in the 6 areas mentioned above. To ensure the high quality of the measurements, the ROI analysis for the quantitative T2 values was performed by a senior expert in musculoskeletal MRI who had no knowledge of the patients.

Second-look arthroscopy was a part of this study protocol. During mosaicplasty and second-look arthroscopy, acoustic evaluation was used to assess cartilage mechanical properties including stiffness, 


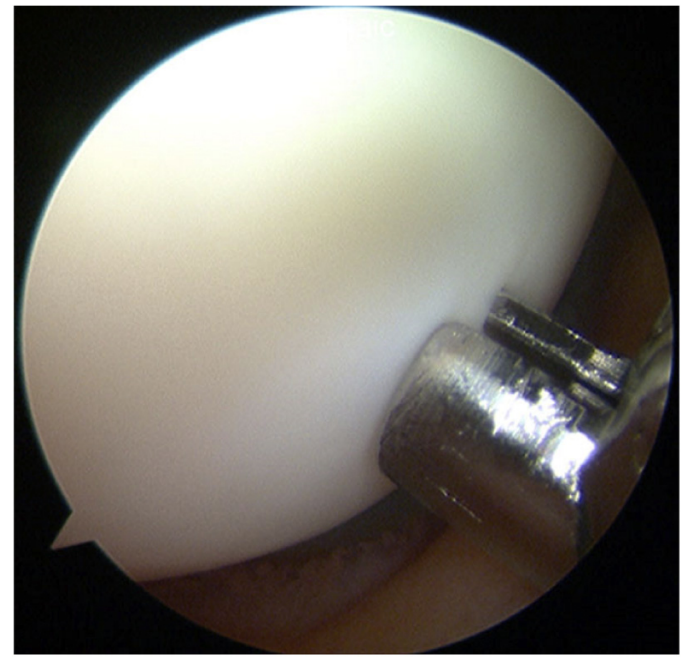

Fig 2. Measurement of cartilage mechanical properties using arthroscopic ultrasonic probe. The mechanical properties of the lateral femoral condylar cartilage were evaluated using the ultrasonic arthroscopic probe. The probe was placed perpendicular to the measured cartilage surface because we could change the angle of the tip of its probe at 5 grades from $0^{\circ}$ to $90^{\circ}$.

surface roughness and thickness of the surrounding knee cartilage, not the donor or recipient sites. The ultrasound assessment systems we used provide quantitative information on tissue properties. ${ }^{18-21}$ Briefly, the system developed by Mori and coworkers ${ }^{21}$ consists of a transducer and pulser/receiver (Panametrics Japan, Tokyo, Japan), digital oscilloscope, and personal computer. The diameter of the transducer, such as an arthroscopic ultrasonic probe, was approximately $3 \mathrm{~mm}$. The central frequency of the ultrasound wave was $10 \mathrm{MHz}$. As the wave passes through interfaces between media of different acoustic impedances, reflections return to the transducer and generate electrical signals in the transducer that are proportional to the intensity. ${ }^{22}$ The validation study for this method was reported by Mori et al. ${ }^{21}$ Fig 2 presents the scene of measurement of cartilage mechanical properties using an arthroscopic ultrasonic probe; the probe was placed perpendicular to the measured cartilage surface because we could change the angle of the tip of its probe at 5 grades from $0^{\circ}$ to $90^{\circ}$. We measured several points which appeared normal or nearly normal cartilage. We calculated the mean values for the cartilage mechanical properties measured in the whole knee joints (W) and lateral compartment of the femur $(\mathrm{L})$. The cartilage in the lateral compartment of the femur in most cases in this study observed as normal or nearly normal with respect to the ICRS classification. Therefore each patient presented a mean value for stiffness, surface roughness, and thickness in the $\mathrm{W}$ or $\mathrm{L}$ at each time point, that is, mosaicplasty or second-look arthroscopy.
The mean values in the $\mathrm{W}$ or $\mathrm{L}$ were compared between the 2 groups at mosaicplasty and second-look arthroscopy. The means of the measured data points with the probe per patient at mosaicplasty were 13.6 points in the $\mathrm{W}$ and 4.3 points in the L; these values at secondlook arthroscopy were 7.7 points in the $\mathrm{W}$ and 2.1 points in the L.

Two-sample 1-sided and paired $t$-tests were used to perform the statistical analysis of the baseline characteristics of the study subjects; blood concentration of curcumin; and the VAS, JKOM, JOA, T2 mapping and cartilage mechanical properties scores. The level of statistical significance was set to a $P$ value $<.05$.

\section{Results}

The baseline characteristics of the study subjects in the two groups are presented in Table 2. There were 14 men and 29 women, and 24 right and 19 left knees. The mean operative age was 59.5 years (range, 24-84 years). Posterior cruciate ligament reconstruction in 1 case (in the placebo group). There were 25 osteoarthritis cases, 10 osteonecrosis cases, and 8 cartilage injury cases. The recipient sites were shown in Table 2 . No statistical differences in the baseline characteristics were evident. Compliance of drugs was similar between the Theracurmin and placebo groups.

Table 2. Comparison of the Characteristics Between the 2 Groups

\begin{tabular}{|c|c|c|c|}
\hline & $\begin{array}{c}\text { Theracurmin } \\
\text { Group } \\
\text { (23 cases) }\end{array}$ & $\begin{array}{c}\text { Placebo } \\
\text { Group } \\
\text { (20 cases) } \\
\end{array}$ & $\begin{array}{c}P \\
\text { values } \\
\end{array}$ \\
\hline $\begin{array}{l}\text { Sex: Male/Female } \\
\quad \text { (cases) }\end{array}$ & $9 / 14$ & $5 / 15$ & .5044 \\
\hline Side: Right/Left (cases) & $15 / 8$ & $9 / 11$ & .3026 \\
\hline Operative age $(\mathrm{y})$ & 58.5 & 60.1 & .5902 \\
\hline Height $(\mathrm{cm})$ & 161.1 & 157.6 & .2579 \\
\hline Weight (kg) & 67.6 & 66.2 & .7661 \\
\hline Recipient area $\left(\mathrm{mm}^{2}\right)$ & 371.8 & 438.8 & .2346 \\
\hline Grafted plugs & 2.8 & 2.8 & .8784 \\
\hline Combined HTO cases & 15 cases $(65.2 \%)$ & 16 cases $(80 \%)$ & .4562 \\
\hline Basic diseases (cases) & & & .5839 \\
\hline Osteoarthritis & 12 & 13 & \\
\hline Osteonecrosis & 5 & 5 & \\
\hline Cartilage injury & & 2 & \\
\hline Recipient sites (cases) & & & .2020 \\
\hline MFC & 16 & 18 & \\
\hline LFC & 1 & 0 & \\
\hline MTP & 1 & 0 & \\
\hline LTP & 1 & 1 & \\
\hline Trochlea & 2 & 0 & \\
\hline MFC + Trochlea & 2 & 0 & \\
\hline $\mathrm{MFC}+\mathrm{MTP}$ & 0 & 1 & \\
\hline
\end{tabular}


Table 3. Serious Adverse Events (Continuous Cases) in the 2 Groups

\begin{tabular}{ll}
\hline \multicolumn{1}{c}{ Case No. } & \multicolumn{1}{c}{ Adverse Event } \\
\hline Theracurmin group & Cholecystitis \\
28 & Fracture \\
29 & Brain infarction \\
32 & Dermatitis \\
39 & Infection \\
43 & Gastric cancer and urethral stone \\
46 & \\
Placebo group & Infection \\
14 & Infection \\
34 & Pecided the above serious \\
\hline
\end{tabular}

The safety committee of this study decided that all the above serious adverse events were not related to curcumin and placebo. If the same patients experienced 2 adverse events, we unblinded the treatment allocation (curcumin or placebo) and decided that the patient should drop out of this study. However, patient 46 wanted to continue this study, and the safety committee approved his continuation in this study.

Serious adverse events in continuous cases are presented in Table 3. There were seven events in six cases in the Theracurmin group and two events in two cases in the placebo group. The safety committee of this study decided that all the serious adverse events shown in Tables 1 and 3 were not related to Theracurmin and the placebo. If the same patients experienced two adverse events, we un-blinded the treatment allocation (Theracurmin or placebo) and decided that the patient should drop out in this study. However, patient 46 wanted to continue this study, and the safety committee approved his continuation in this study. Abnormal blood biochemistry values before mosaicplasty and 12 months after mosaicplasty were compared between the 2 groups (Table 4). There were 9 abnormal values among 6 cases in the Theracurmin group and 8 among seven cases in the placebo group. There were no significant differences in the number of abnormal values between the two groups. Therefore, in this study, the occurrence ratio of the adverse events and abnormal blood biochemistry values in the Theracurmin group was similar to that in the placebo group. We

Table 4. Comparison of Abnormal Blood Biochemistry Values Before Mosaicplasty and 12 Months After Mosaicplasty Between the 2 Groups

\begin{tabular}{lll}
\hline & $\begin{array}{c}\text { Theracurmin group } \\
(23 \text { cases })\end{array}$ & $\begin{array}{c}\text { Placebo group } \\
(20 \text { cases })\end{array}$ \\
\hline Triglyceride increase & 4 cases & 1 case \\
Total cholesterol increase & 1 case & 3 cases \\
Choline esterase decrease & 1 case & 1 case \\
Eosinophil increase & 1 case & 1 case \\
Red blood cell increase & 0 case & 1 case \\
Red blood cell decrease & 1 case & 0 case \\
Amylase decrease & 1 case & 0 case \\
Creatinine increase & 0 case & 1 case \\
\hline
\end{tabular}

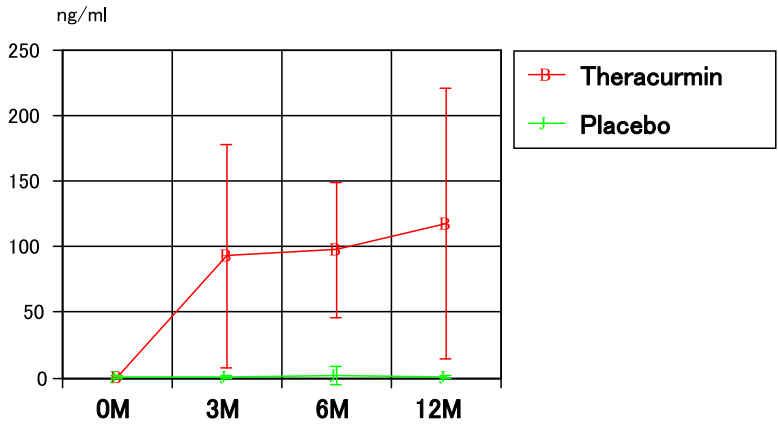

Fig 3. The blood concentrations of curcumin in the two groups.

demonstrated that the oral administration of Theracurmin for 1 year was safe.

The blood concentrations of curcumin in the two groups are presented in Fig 3; the values in the Theracurmin group at 3, 6, and 12 months after mosaicplasty were significantly higher than those in the placebo group. The JOA scores for the 2 groups are presented in Fig 4. There were no significant differences between the groups with respect to the JOA scores before and at 3, 6, and 12 months after mosaicplasty. The JOA scores at 3 , 6 , and 12 months after mosaicplasty in both groups were significantly higher than those before mosaicplasty in both groups. Similarly, between the two groups, there were no significant differences in the VAS and JKOM scores before mosaicplasty and 3, 6, and 12 months after mosaicplasty. The VAS and JKOM scores at 3, 6, and 12 months after mosaicplasty in both groups were significantly higher than those before mosaicplasty in both groups.

Fig 5 presents the chronological changes in T2 mapping values in the lateral tibia for the 2 groups. There were no significant differences in these values at each time point between the 2 groups, and the values for each group before mosaicplasty were not significantly different from those at 3, 6, and 12 months after mosaicplasty. Similarly, in the other 5 sites, there were no significant in these values at each time point between the 2 groups.

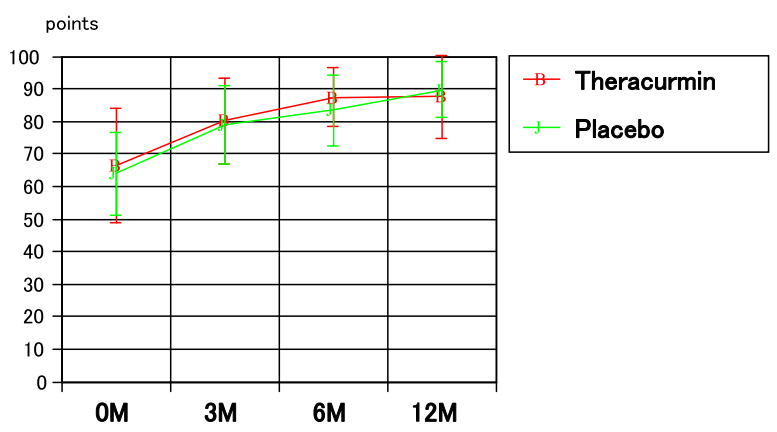

Fig 4. The JOA scores in the two groups. 


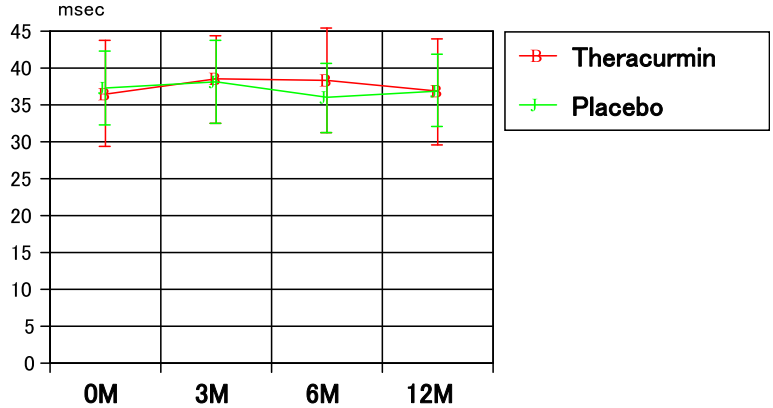

Fig 5. The chronological changes in $\mathrm{T} 2$ mapping values in the lateral tibia in the two groups.

The mean time from the mosaicplasty to the secondlook arthroscopy was 13.9 months (range, 12-23 months). The mean time in the Theracurmin group was 14.0 months (range, 12-23 months), and that in the placebo group was 13.7 months (range 12-18 months); there were no significant differences. The mean values for stiffness (relative value) in the $\mathrm{W}$ at mosaicplasty in the Theracurmin group were not significantly different from those in the placebo group $(P=.4295)$ (Fig 6A). However, the value in the $\mathrm{W}$ at second-look arthroscopy in the Theracurmin group was significantly better than that in the placebo group $(P=.0261)$ (Fig 6B). The mean values for surface roughness in the $\mathrm{L}$ at mosaicplasty in the Theracurmin group were not significantly different from those in the placebo group $(P=.5049)$ (Fig 6C). However, values in the $\mathrm{L}$ at second-look arthroscopy in the Theracurmin group were significantly better than those in the placebo group $(P=.0499)$ (Fig 6D). The other mean values for the mechanical properties of the cartilage at mosaicplasty or second-look arthroscopy were not significantly different between the 2 groups.

\section{Discussion}

In this study, there were no significant differences in the occurrence ratio of adverse events and abnormal blood biochemistry values between the Theracurmin group and the placebo group. In the 2 groups, there were no significant differences in the JOA, VAS, and JKOM scores before and 3, 6, and 12 months after mosaicplasty. No significant differences were observed in the T2 mapping values at each time point between the two groups. The blood concentrations of curcumin
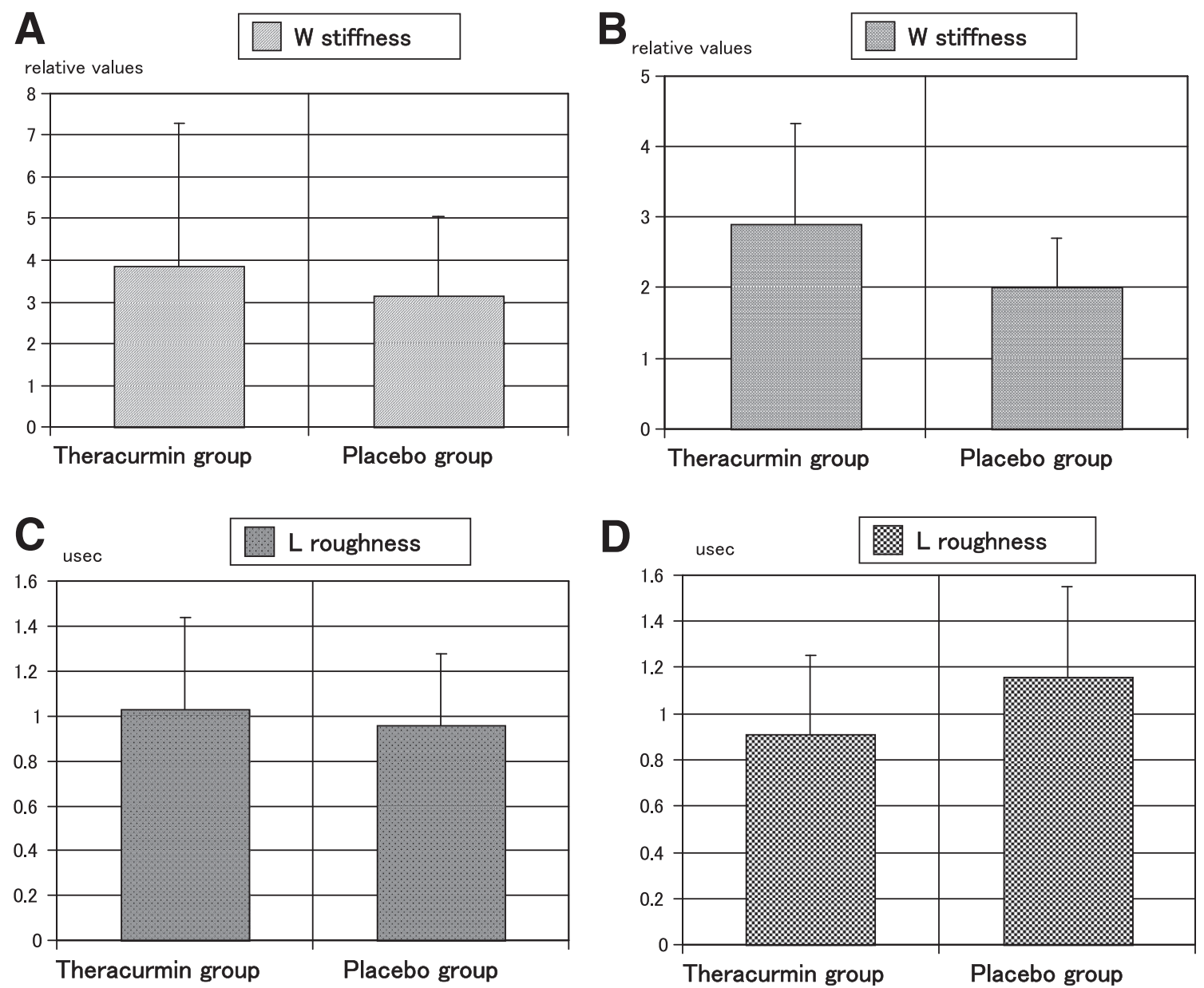

Fig 6. The mean values for the mechanical properties of the knee cartilage. 
in the Theracurmin group at 3, 6, and 12 months after mosaicplasty were significantly higher than those in the placebo group. The mean value for stiffness in the $\mathrm{W}$ at second-look arthroscopy in the Theracurmin group was significantly better than that in the placebo group. The mean value for the surface roughness in the $\mathrm{L}$ at second-look arthroscopy in the Theracurmin group was significantly better than that in the placebo group. The oral administration of Theracurmin for 1 year demonstrated significantly better chondroprotective effects and no worse clinical effects and adverse events than the placebo.

In the clinical use of curcumin, Nakagawa et al. ${ }^{12,13}$ demonstrated the anti-inflammatory effect of Theracurmin. In a randomized open-label parallel-arm study, patients receiving curcumin showed similar improvement in the severity of pain and the Knee Injury and Osteoarthritis Outcome Score compared with those receiving diclofenac at days 14 and $28 .^{23}$ In double-blind randomized placebo controlled studies, Henrotin reported that pain reduction at day 90 in groups administered low- and high-dose bio-optimized Curcuma longa extract was higher than that in the placebo group ${ }^{24}$; Hashemzadeh et al. ${ }^{25}$ stated that patients administered nano curcumin exhibited a significant decrease in the overall score, as well as the scores or the pain, stiffness, and physical activity subscales of the WOMAC questionnaire, compared with the placebo group.

Several studies have demonstrated the chondroprotective effects of curcumin in cell culture or a rat osteoarthritis model. Csaki et al. ${ }^{26}$ reported that treatment with curcumin and resveratrol suppressed NF-kBregulated gene products involved in inflammation, inhibited apoptosis and prevented caspase- 3 activation in cell culture. Wang et al. ${ }^{27}$ revealed that curcumin decreased MMP-13 expression and increased type II collagen expression in IL-lb-stimulated chondrocytes. A complex of curcumin and lecithin that was developed to improve the solubility of curcumin enhanced the chondroprotective effect of curcumin via an inflammatory mechanism in cell culture. ${ }^{28}$ In a rat osteoarthritis model, Wang et al. $^{29}$ demonstrated that hyaluronic acid/chitosan nanoparticles for the delivery of curcuminoids may suppress inflammation and chondrocyte apoptosis in knee osteoarthritis via repression of the NF-kB pathway. Theracurmin treatment significantly ameliorated cartilage damage and chondrocyte decrease in rats with monosodium iodoacetate-induced osteoarthritis. ${ }^{30}$ However, to date, no studies have demonstrated the chondroprotective effects of Theracurmin or curcumin in a human clinical trial.

Quantitative T2 mapping is a noninvasive technique used clinically to measure knee cartilage characterization. In young professional soccer players, the T2 relaxation times are significantly increased in the weight-bearing femoral cartilage of the medial and lateral compartment of the knee when comparing early unloading with late unloading. ${ }^{31}$ Early degeneration could occur in various knee cartilage compartments after acute anterior cruciate ligament rupture, especially in the superficial layer of the lateral tibia in T2 mapping. ${ }^{32}$ Unfortunately, the present study demonstrated no significant differences in $\mathrm{T} 2$ mapping values at each time point between the 2 groups. Presumably 1 year may be too early a time point to measure significant cartilage degeneration.

Recently, 2 studies evaluated the mechanical properties of articular cartilages. Pulse-echo ultrasound geometry at $5 \mathrm{MHz}$ was feasible for simultaneous measurement of the acoustic properties of the articular cartilage and subchondral bone. ${ }^{33}$ Stress relaxation optical coherence straingraphy provided important data to understand the biomechanics of early osteoarthritis. ${ }^{34}$ As previously described, Mori et al. ${ }^{21}$ developed a device to measure the mechanical properties of the articular cartilages via ultrasound. ${ }^{18}$ Several studies that used this device reported significant results for the mechanical properties of the articular cartilages in humans and rabbits. ${ }^{19,20,35-37}$ Hattori et al. ${ }^{38}$ reported the acoustic properties of living human cartilage differ between the knee and ankle joints, as observed using his arthroscopic ultrasonic probe and Mori's system. Using Mori's system and our arthroscopic ultrasonic probe, our present study (Fig 2) demonstrated that the mean values for stiffness (relative value) in the $\mathrm{W}$ and surface roughness in the $\mathrm{L}$ at mosaicplasty in the Theracurmin group was not significantly different from that in the placebo group (Figs. 6a and 6c). However, stiffness in the $\mathrm{W}$ and surface roughness in the $\mathrm{L}$ at second-look arthroscopy in the Theracurmin group was significantly better than that in the placebo group (Figs. $6 \mathrm{~b}$ and $6 \mathrm{~d}$ ). These data may be affected by high tibial osteotomy, but the ratio of high tibial osteotomy was not significant in 2 groups. There were no significant differences in cartilage thickness between the 2 groups in our study. These results were similar to those of Nicoliche's study, which demonstrated no increase in cartilage thickness in the knees of rats with induced arthritis that were treated with curcumin. ${ }^{39}$ Kuroki et al. $^{35}$ revealed that modified Mankin's score was significantly correlated with stiffness and surface roughness. The present study demonstrates the chondroprotective effect of curcumin in a clinical trial. We think that the mechanical properties of the articular cartilages are more sensitive than T2 mapping in the cartilage characteristics. Our present study provides early evidence that Theracurmin may provide chondroprotective effects, but longer-term data will be needed to conform continued protection from degeneration. 
In the present study, 16 serious adverse events were detected among 12 cases (Tables 1 and 3); however, the safety committee of this study decided that all serious adverse events were not related to Theracurmin and the placebo. There were 17 abnormal blood biochemistry values among 13 cases in the present study; however, the occurrence ratio of the adverse events and the abnormal blood biochemistry values in the Theracurmin group were similar to those in the placebo group. Previously, 2 studies by Nakagawa et al. ${ }^{12,13}$ using Theracurmin exhibited no major side effects. ${ }^{12,13}$ Similarly, no considerable adverse effects were observed with curcumin administration in other clinical studies. ${ }^{40-43}$ Curcuma domestica extracts were noninferior to ibuprofen for the treatment of knee osteoarthritis, and the incidences of adverse events were similar for both; however, gastrointestinal adverse events were observed with the C. domestica extracts. ${ }^{44}$ Wu et al. ${ }^{45}$ reported that the side effect rate of curcumin treatment was 0.81 times higher than that of ibuprofen treatment. Gupte et al. ${ }^{46}$ revealed that intake of solid lipid curcumin particles for 90 days demonstrated no change in the hemogram and biochemical markers. Daily reported that turmeric preparations and curcumin were considered safe at doses not exceeding $1200 \mathrm{mg} / \mathrm{d}$ for up to 4 months. ${ }^{47}$ A study on the safety of Theracurmin revealed that a dose of up to $400 \mathrm{mg}$ for 9 months was safe in cancer patients receiving chemotherapy. ${ }^{48}$ In our present study, the dosage of curcumin was $180 \mathrm{mg} / \mathrm{d}$, similar to that used in Nakagawa's studies. ${ }^{12,13}$ This is the lowest dose used in published clinical studies. ${ }^{49}$ The safety of curcumin was demonstrated in our present study, and the dosage of curcumin used in our study was safe.

\section{Limitations}

The limitations of this study are the small samples size (50 cases), short follow-up periods (12 months), and various basic diseases and operative ages.

\section{Conclusions}

The oral administration of Theracurmin for 1 year demonstrated significantly better chondroprotective effects and no worse clinical effects and adverse events than the placebo.

\section{Acknowledgments}

We thank Mrs. Kaori Akiyoshi for technical assistance and Mr. Jun Kumakyu for assisting with MRI T2 mapping.

\section{References}

1. Goel A, Kunnumakkara AB, Aggarwal BB. Curcumin as "Curecumin": From kitchen to clinic. Biochem Pharmacol 2008;75:787-809.
2. Gupta SC, Patchva S, Koh W, Aggarwal BB. Discovery of curcumin, a component of golden spice, and its miraculous biological activities. Clin Exp Pharmacol Physiol 2012;39:283-299.

3. Menon VP, Sudheer AR. Antioxidant and antiinflammatory properties of curcumin. Adv Exp Med Biol 2007;595:105-125.

4. Rao CV. Regulation of COX and LOX by curcumin. Adv Exp Med Biol 2007;595:213-226.

5. Milacic V, Banerjee S, Landis-Piwowar KR, Sarkar FH, Majumbar AP, Dou QP. Curcumin inhibits the proteasome activity in human colon cancer cells in vitro and in vivo. Cancer Res 2008;68:7283-7292.

6. Buhrmann C, Mobasheri A, Matis U, Shakibaei M. Curcumin mediated suppression of nuclear factor-kB promotes chondrogenic differentiation of mesenchymal stem cells in a high-density co-culture microenvironment. Arthritis Res Ther 2010;12:R127.

7. Mathy-Hartert M, Jacquemond-Collet I, Priem F, Sanchez C, Lambert C, Henrotin Y. Curcumin inhibits pro-inflammatory mediators and metalloproteinase-3 production by chondrocytes. Inflamm Res 2009;58: 899-908.

8. Wongcharoen $\mathrm{W}$, Phrommintikul A. The protective role of curcumin in cardiovascular diseases. Int $J$ Cardiol 2009;133:145-151.

9. Shakibaei M, Schulze-Tanzil G, John T, Mobasheri A. Curcumin protects human chondrocytes from IL-1binduce inhibition of collagen type II and betal-integrin expression and activation of caspase-3: an immunomorphological study. Ann Anat 2005;187:487-497.

10. Rajasekaran SA. Therapeutic potential of curcumin in gastrointestinal diseases. World J Gastrointest Pathophysiol $2011 ; 15: 1-14$.

11. Sasaki H, Sunagawa Y, Takahashi K, et al. Innovative preparation of curcumin for improved oral bioavailability. Biol Pharm Bull 201 1;34:660-665.

12. Nakagawa Y, Mukai S, Yamada S, et al. Short-term effects of highly-bioavailable curcumin for treating knee osteoarthritis: A randomized, double-blind, placebo-controlled prospective study. J Orthop Sci 2014;19:933-939.

13. Nakagawa Y, Mukai S, Yamada S, et al. The efficacy and safety of highly-bioavailable curcumin for treating knee osteoarthritis-A six-month study. Clin Med Insights Arthritis Musculoskelet Disord 2020;13:1 179544120948471.

14. Akai M, Doi T, Fujino K, Iwaya T, Kurosawa H, Nasu T. An outcome measure for Japanese people with knee osteoarthritis. J Rheum 2005;32:1524-1532.

15. Okuda M, Omokawa S, Okahashi K, Akahane M, Tanaka Y. Validity and reliability of the Japanese Orthopaedic Association score for osteoarthritic knees. J Orthop Sci 2012;17:750-756.

16. Domayer SE, Kutscha-Lissberg F, Welsch G, et al. T2 mapping in the knee after microfracture at 3.0T: Correlation of global T2 values and clinical outcomepreliminary results. Osteoarthr Cartil 2008;16:903-908.

17. Kuo CK, Li WJ, Mauck RL, Tuan RS. Cartilage tissue engineering: its potential and uses. Curr Opin Rheumatol 2006;18:64-73.

18. Hattori K, Mori K, Habata T, Takakura Y, Ikeuchi K. Measurement of the mechanical condition of articular 
cartilage with an ultrasonic probe: quantitative evaluation using wavelet transformation. Clin Biomech 2003;18: 553-557.

19. Kuroki H, Nakagawa Y, Mori K, Ikeuchi K, Nkamura T. Mechanical effects of autologous osteochondral surgical grafting procedures and instrumentation on grafts of articular cartilage. Am J Sport Med 2004;32:612-620.

20. Kuroki H, Nakagawa Y, Mori K, et al. Maturationdependent change and regional variations in acoustic stiffness of rabbit articular cartilage: An examination of the superficial collagen-rich zone of cartilage. Osteoarthritis Cartilage 2006;14:784-792.

21. Mori K, Hattori K, Habata T, et al. Measurement of the mechanical properties of regenerated articular cartilage using wavelet transformation. In: Ikada Y, Umakoshi Y, Hotta T, eds. Tissue Engineering for Therapeutic Use. Tokyo: Elsevier, 2002;133-142.

22. Rushfeldt PD, Mann RW, Harris WH. Improved techniques for measuring in vitro the geometry and pressure distribution in the human acetabulum-I. Ultrasonic measurement of acetabular surfaces, sphericity and cartilage thickness. J Biomech 1981;14:253-260.

23. Shep D, Khanwelkar C, Gade P, Karad S. Safety and efficacy of curcumin versus diclofenac in knee osteoarthritis: A randomized open-label parallel-arm study. Trials 2019;20:214.

24. Henrotin Y, Malaise M, Wittoek R, et al. Bio-optimized curcuma longa extract is efficient on knee osteoarthritis pain: A double-blind multicenter randomized placebo controlled three-arm study. Arthritis Res Ther 2019;21: 179- 187.

25. Hashemzadeh K, Davoudian N, Jaafari MR, Mirfeizi Z. The effect of nanocurcumin in improvement of knee osteoarthritis: A randomized clinical trial. Curr Rheumatol Rev 2020;16:158-164.

26. Csaki C, Mobasheri A, Shakibaei M. Synergistic chondroprotective effects of curcumin and resveratrol in human articular chondrocytes: Inhibition of IL-1b-induced NF-kB-mediated inflammation and apoptosis. Arthritis Res Ther 2009;11:R165.

27. Wang J, Ma J, Gu JH, et al. Regulation of type II collagen, matrix metalloproteinase-13 and cell proliferation by interleukin-1b is mediated by curcumin via inhibition of NF-kB signaling in rat chondrocytes. Mol Med Rep 2017;16: 1837-1845.

28. Kim L, Kim JY. Chondroprotective effect of curcumin and lecithin complex in human chondrocytes stimulated by IL-1b via an anti-inflammatory mechanism. Food Sci Biotechnol 2019;28:547-553.

29. Wang J, Wang X, Cao Y, Huang T, Song DX, Tao HR. Therapeutic potential of hyaluronic acid/chitosan nanoparticles for the delivery of curcuminoid in knee osteoarthritis and an in vitro evaluation in chondrocytes. Int $J$ Mol Med 2018;42:2604-2614.

30. Park HJ, Lee CK, Song SH, Yun JH, Lee A, Park HJ. Highly bioavailable curcumin powder suppresses articular cartilage damage in rats with mono-iodoacetate (MIA)-induced osteoarthritis. Food Sci Biotechnol 2019;29:251-263.

31. Wldenmeier L, Evers C, Uder M, et al. Using cartilage MRI T2-mapping to analyze early cartilage degeneration in the knee joint of young professional soccer players. Cartilage 2019;10:288-298.

32. Tao H, Qiao Y, Hu Y, et al. Quantitative T2-mapping and $\mathrm{T} 2 *$-mapping evaluation of changes in cartilage matrix after acute anterior cruciate ligament rupture and the correlation between the results of both methods. Biomed Res Int 2018;2018:7985672.

33. Aula AS, Toyras J, Tiitu V, Jurvelin JS. Simultaneous ultrasound measurement of articular cartilage and subchondral bone. Osteoarthritis Cartilage 2010;18: 1570-1576.

34. Nakamura S, Ikebuchi M, Saeki S, et al. Changes in viscoelastic properties of articular cartilage in early stage of osteoarthritis, as determined by optical coherence tomography-based strain rate tomography. BMC Musculoskel Disord 2019;20:417.

35. Kuroki H, Nakagawa Y, Mori K, et al. Acoustic stiffness and change in plug cartilage over time after autologous osteochondral grafting: correlation between ultrasound signal intensity and histological score in a rabbit model. Arthritis Res Ther 2004;6:R492-R504.

36. Kuroki H, Nakagawa Y, Mori K, et al. Sequential changes in implanted cartilage after autologous osteochondral transplantation: Postoperative acoustic properties up to 1 year in an in vivo rabbit model. Arthroscopy 2007;23: 647-654.

37. Kuroki H, Nakagawa Y, Mori K, et al. Ultrasound properties of articular cartilage in the tibio-femoral joint in knee osteoarthritis: Relation to clinical assessment (International Cartilage Repair Society grade). Arthritis Res Ther 2008;10:R78.

38. Hattori K, Takakura Y, Ishimura M, Tanaka Y, Habata T, Ikeuchi K. Differential acoustic properties of early cartilage lesions in living human knee and ankle joints. Arthritis Rheum 2005;52:3125-3131.

39. Nicoliche T, Maidonado DC, Faber J, Silva MCPd. Evaluation of the articular cartilage in the knees of rats with induced arthritis treated with curcumin. Plos One 2020;15: e0230228.

40. Appelboom T, Maes N, Albert A. A new Curcuma extract (Flexofytol) in osteoarthritis: Results from a Belgian reallife experience. Open Rheum J 2014;8:77-81.

41. Haroyan A, Mukuchyan V, Mkrtchyan N, et al. Efficacy and safety of curcumin and its combination with boswellic acid in osteoarthritis: A comparative, randomized, doubleblinded, placebo-controlled study. BMC Complement Alternat Med 2018;18:7.

42. Panahi Y, Rahimnia AR, Sharafi M, Alishiri G, Saburi A, Sahebkar A. Curcuminoids treatment for knee osteoarthritis: A randomized double-blind placebo-controlled trial. Phytother Res 2014;28:1625-1631.

43. Sterzi S, Giordani L, Morrone M, et al. The efficacy and safety of a combination of glucosamine hydrochloride, chondroitin sulfate and bio-curcumin with exercise in the treatment of knee osteoarthritis: A randomized, doubleblind, placebo-controlled study. Eur J Phys Rehabil Med 2016;52:321-330.

44. Kuptniratsaikul V, Dajpratham P, Buntragulpoontawee M, et al. Efficacy and safety of Curcuma domestica extracts compared with ibuprofen in patients with knee 
osteoarthritis: A multicenter study. Clin Interv Aging 2014;9: 451-458.

45. Wu J, Lv M, Zhou Y. Efficacy and side effect of curcumin for the treatment of osteoarthritis: A meta-analysis of randomized controlled trials. Pak J Pharm Sci 2019;32: 43-51.

46. Gupte PA, Giramkar SA, Harke SM, et al. Evaluation of the efficacy and safety of capsule Longvida optimized curcumin (solid lipid curcumin particles) in knee osteoarthritis: A pilot clinical study. J Inflam Res 2019;1: 145-152.
47. Daily JW, Yang M, Park S. Efficacy of turmeric extracts and curcumin for alleviating the symptoms of joint arthritis: A systematic review and meta-analysis of randomized clinical trials. J Med Food 2016;19:717-729.

48. Kanai M, Otsuka Y, Otsuka K, et al. A phase I study investigating the safety and pharmacokinetics of highly bioavailable curcumin (Theracurmin) in cancer patients. Cancer Chemother Pharmacol 2013;71:1521-1530.

49. Chin KY The spice for joint inflammation: antiinflammatory role of curcumin in treating osteoarthritis. Drug Design Develop Ther 2016;10:3029-3042. 